\title{
Values of Apparent Diffusion Coefficient and Lesion-to-Spinal Cord Signal Intensity in Diagnosing Solitary Pulmonary Lesions: Turbo Spin-Echo versus Echo-Planar Imaging Diffusion- Weighted Imaging
}

\author{
Qiang Lei ${ }^{1},{ }^{1}$ Qi Wan, ${ }^{2}$ Lishan Liu, ${ }^{3}$ Jianfeng Hu, ${ }^{2}$ Wei Zuo, ${ }^{1}$ Jianneng Li, ${ }^{1}$ Guihua Jiang, \\ and Xinchun $\mathrm{Li} \mathbb{D}^{2}$ \\ ${ }^{1}$ Department of Medical Imaging, Guangdong Second Provincial General Hospital, Shiliugang Rd, Haizhu District Guangzhou, \\ China 510317 \\ ${ }^{2}$ Department of Radiology, The First Affiliated Hospital of Guangzhou Medical University, 151 Yanjiangxi Road, Guangzhou, China \\ 510120 \\ ${ }^{3}$ Department of Radiology, The Fifth Affiliated Hospital of Guangzhou Medical University, 621 Gangwan Road, Guangzhou, China \\ 510799 \\ ${ }^{4}$ Department of Medical Imaging, Guangdong Second Provincial General Hospital, Shiliugang Rd, Haizhu District Guangzhou, \\ China 510317
}

Correspondence should be addressed to Xinchun Li; xinchunli@163.com

Received 22 May 2021; Revised 30 June 2021; Accepted 12 July 2021; Published 16 August 2021

Academic Editor: Marco Giannelli

Copyright ( 2021 Qiang Lei et al. This is an open access article distributed under the Creative Commons Attribution License, which permits unrestricted use, distribution, and reproduction in any medium, provided the original work is properly cited.

Objective. This study is aimed at comparing the image quality and diagnostic performance of mean apparent diffusion coefficient (ADC) and lesion-to-spinal cord signal intensity ratio (LSR) derived from turbo spin-echo diffusion-weighted imaging (TSE-DWI) and echo-planar imaging- (EPI-) DWI in patients with a solitary pulmonary lesion (SPL). Methods. 33 patients with SPL underwent chest imaging using EPI-DWI and TSE-DWI with $b=600 \mathrm{~s} / \mathrm{mm}^{2}$ in free breathing. A comparison of the distortion ratio (DR), signal-to-noise ratio (SNR), and contrast-to-noise ratio (CNR) was drawn between the two techniques using a Wilcoxon signed-rank test. The interprotocol reproducibility between quantitative parameters of EPI-DWI and TSE-DWI was evaluated using a Bland-Altman plot. ADCs and LSRs derived from EPI-DWI and TSE-DWI were calculated and compared between malignant and benign groups using the Mann-Whitney test. Results. TSE-DWI had similar SNR and CNR compared with EPI-DWI. DR was significantly lower on TSE-DWI than EPI-DWI. ADC and LSR showed slightly higher values with TSE-DWI, while the Bland-Altman analysis showed unacceptable limits of agreement between the two sequences. ADC and LSR of both DWI techniques differed significantly between lung cancer and benign lesions $(P<0.05)$. The $\operatorname{LSR}_{(\mathrm{EPI}-\mathrm{DWI})}$ showed the highest area under the curve $(\mathrm{AUC}=0.818)$, followed by $\mathrm{ADC}_{(\mathrm{EPI}-\mathrm{DWI})}$ $(\mathrm{AUC}=0.789), \mathrm{ADC}_{(\mathrm{TSE}-\mathrm{DWI})}(\mathrm{AUC}=0.781)$, and $\mathrm{LSR}_{(\mathrm{TSE}-\mathrm{DWI})}(\mathrm{AUC}=0.748)$, respectively. Among these parameters, the difference in diagnostic accuracy was not statistically significant. Conclusions. TSE-DWI provides significantly improved image quality in patients with SPL as compared with EPI-DWI. However, there was no difference in diagnostic efficacy between these two techniques, according to ADC and LSR. 


\section{Introduction}

Diffusion-weighted imaging (DWI) is an essential magnetic resonance imaging (MRI) technique to assess water molecule diffusivity in living tissues. Single-shot echo-planar imaging (SS-EPI) is the most used DWI technique because of its short acquisition time [1]. However, the image quality of the standard SS-EPI-DWI is frequently deteriorated by magnetic inhomogeneity, as EPI acquisition is prone to phase error accumulation [2].

In the lung, magnetic inhomogeneity exists at the airlung interface, leading to signal loss or image distortion in EPI-DWI, which might hamper accurate lesion measurements of the derived parameters [3]. Turbo spinecho (TSE-) DWI might be an excellent alternative to EPI-DWI for patients sensitive to image distortion [4-6]. In recent years, studies [7-9] compared the image quality and the reproducibility of apparent diffusion coefficient (ADC) between EPI-DWI and TSE-DWI in areas such as oral, head, and neck. A recent study has documented the ability of TSE-DWI to improve the image quality and test-retest robustness of ADC and intravoxel incoherent motion (IVIM) parameters in patients with lung cancer [10]. However, it is unclear whether there is any diagnostic difference between these two sequences in assessing solitary pulmonary lesions.

Previous studies suggested that ADC could be a useful parameter in distinguishing malignant from benign pulmonary lesions [11]. On the other hand, some studies demonstrated that the lesion-to-spinal cord signal intensity ratio (LSR), as a semiquantitative method, has better diagnostic efficacy than ADC [12]. However, other studies reported inconsistent findings [13-15].

Therefore, the purpose of this study was to compare the image quality as well as the diagnostic performance of ADC and LSR derived from EPI-DWI and TSE-DWI in patients with solitary pulmonary lesions (SPLs).

\section{Materials and Methods}

2.1. Patient Population. This study was approved by the institutional review board, and written informed consent was obtained from each patient with a solitary pulmonary nodule or masses confirmed by computed tomography (CT) findings. The inclusion criteria were as follows: (a) lesions were measurable on CT images; (b) there were no contraindication for MRI examinations; and (c) patients did not undergo any therapies. A total of 33 patients (21 men and 12 women; age range, 33-77 years; mean age, 57 years) underwent a DWI scan of the lung. The pathological assessment revealed 22 malignant tumors (15 invasive adenocarcinomas, one squamous carcinoma, three small-cell carcinomas, one lymphoid epithelioid carcinoma, and two mucoepidermoid carcinomas) and 11 benign lesions (one hamartoma, two sclerosing alveolar cell tumors, and eight granulomas).

2.2. Image Acquisition. All patients were examined on a 3.0 T MRI scanner equipped with a 16-channel body phase array coil (Achieva, Philips Healthcare, Best, the
TABLE 1: Scanning parameters of magnetic resonance imaging.

\begin{tabular}{lccc}
\hline & TSE-T2WI & EPI-DWI & TSE-DWI \\
\hline TR (ms) & 973 & 1238 & 5965 \\
TE (ms) & 80 & 51 & 56 \\
NSA & 1 & 4 & 4 \\
FOV (mm) & $430 \times 349$ & $260 \times 423$ & $260 \times 423$ \\
Slice thickness (mm) & 7 & 5 & 5 \\
Gap (mm) & 0.7 & 0.5 & 1 \\
$b$ value (s/mm ${ }^{2}$ ) & - & 600 & 600 \\
Parallel imaging factor & 2 & 3 & 3 \\
Matrix & $360 \times 247$ & $88 \times 140$ & $80 \times 140$ \\
BW (Hz/pixel) & 565.2 & 43.5 & 371.4 \\
Recon voxel size (mm) & 0.67 & 1.1 & 1.1 \\
Scanning time & $23 \mathrm{~s}$ & $36 \mathrm{~s}$ & $2 \mathrm{~min} 47 \mathrm{~s}$ \\
\hline
\end{tabular}

Note: TE: echo time; TR: repetition time; NSA: number of signals acquired; FOV: field of view; m: minutes; s: seconds; BW: bandwidth.

Netherlands). Axial TSE T2-weighted imaging (T2WI) was performed with the following parameters: repetition time (TR), $973 \mathrm{~ms}$; echo time (TE), $80 \mathrm{~ms}$; field of view $($ FOV $), 430 \times 349 \mathrm{~mm}^{2}$; matrix size, $360 \times 247$; and slice thickness, $7 \mathrm{~mm}$, with a $0.7 \mathrm{~mm}$ intersection gap. Both SS-TSE sequence and SS-EPI sequences with $b_{0}$ and $b$ value $600 \mathrm{~s} / \mathrm{mm}^{2}$ were used to acquire DWI images in free breathing. The EPI-DWI's sequence parameters were as follows: FOV, $260 \times 423 \mathrm{~mm}^{2}$; TR/TE, $1238 \mathrm{~ms} / 51 \mathrm{~ms}$; slice thickness, $5 \mathrm{~mm}$; number of signal averages (NSA), 3 ; and acquisition time, $36 \mathrm{~s}$. The TSE-DWI's sequence parameters were as follows: FOV, $260 \times 423 \mathrm{~mm}^{2}$; TR/TE, $5965 \mathrm{~ms} / 56 \mathrm{~ms}$; slice thickness, $5 \mathrm{~mm}$; NSA, 3; and acquisition time, 2 min $47 \mathrm{~s}$ (Table 1).

2.3. Image Quality. The image quality of 33 patients was quantitatively assessed by a radiologist under an experienced radiologist (five years and ten years of magnetic resonance diagnostic practice, respectively). The image distortion along a phase-encoding direction, signal-tonoise ratio (SNR), and contrast-to-noise ratio (CNR) from EPI-DWI and TSE-DWI (with $b=600 \mathrm{~s} / \mathrm{mm}^{2}$ ) were recorded. Regions of interest (ROIs) were manually drawn on the solid part of the lesion at the level of maximum transverse diameter avoiding necrosis and hemorrhage, spinal cord $\left(12-16 \mathrm{~mm}^{2}\right)$, thoracic muscle $\left(40-50 \mathrm{~mm}^{2}\right)$, and background $\left(40-50 \mathrm{~mm}^{2}\right)$ for mean signal intensity (SI) and standard deviation (SD) measurements.

In the RadiAnt DICOM Viewer 4.6.5.18450 (Medixant, Poznan, Poland), the fusion images were generated by superimposing DWI with T2WI in each sequence, and image distortions of the solitary pulmonary nodules or mass were evaluated using the distortion ratio (DR):

$$
\mathrm{DR}=\frac{a^{10}}{b}
$$




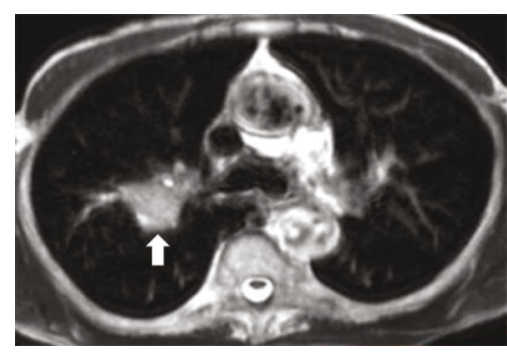

(a)

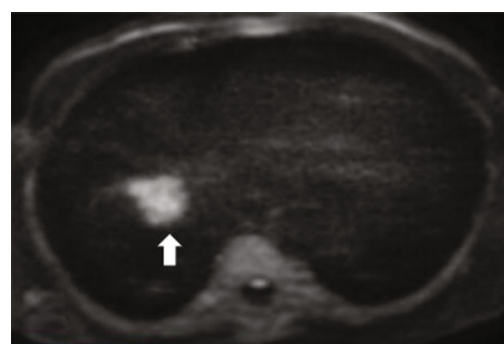

(c)

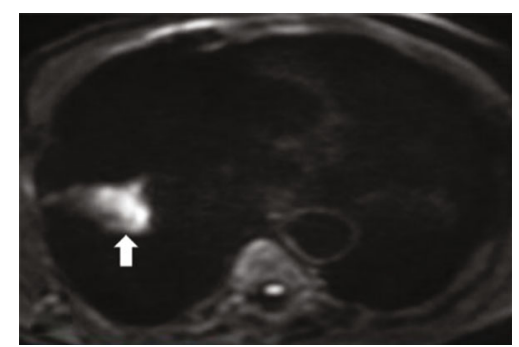

(b)

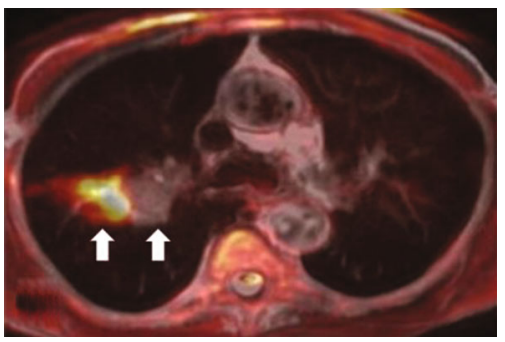

(d)

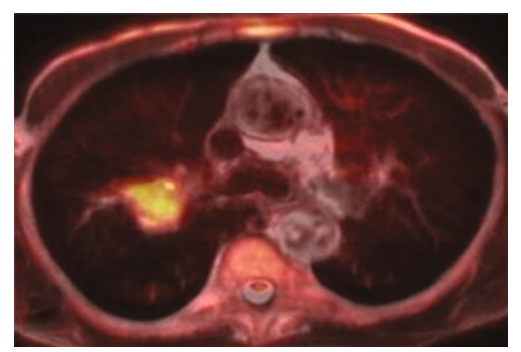

(e)

FIGURE 1: A nodular lesion (arrow) in the right upper lobe: (a) TSE-T2WI; (b) EPI-DWI $\left(b=600 \mathrm{~s} / \mathrm{mm}^{2}\right)$; (c) TSE-DWI ( $\left.b=600 \mathrm{~s} / \mathrm{mm} 2\right)$; (d) T2WI fused with EPI-DWI; (e) T2WI fused with TSE-DWI. The distortion and displacement of the lesion (double fine arrow) are illustrated on EPI-DWI. Fused images demonstrated that TSE-DWI is perfectly matched with T2WI.

where $a$ is the maximum displacement along the phaseencoding path of the lesion between T2WI and the corresponding DWI and $b$ is the diameter along the phaseencoding path of the lesion on T2WI.

SNR, CNR, and LSR were calculated according to the following equations:

$$
\begin{gathered}
\mathrm{SNR}=\frac{\mathrm{SI}_{\text {lesion }}}{\mathrm{SI}_{\text {background }}}{ }^{16}, \\
\mathrm{CNR}=\frac{\mathrm{SI}_{\text {lesion }}-\mathrm{SI}_{\text {muscle }}}{\mathrm{SD}_{\text {background }}}, \\
\mathrm{LSR}=\frac{\mathrm{SI}_{\text {lesion }}}{\mathrm{SI}_{\text {spinal cord }}} .
\end{gathered}
$$

2.4. Image Postprocessing. MRI images were postprocessed using PRIDE software (Philips Healthcare). ADC was generated with $b$ values of 0 and $600 \mathrm{~s} / \mathrm{mm}^{2}$. ROIs were manually drawn in the solid lesion on the slice with a maximum transverse diameter under the guidance of an experienced radiologist, avoiding necrosis and hemorrhage.
The ADC value was calculated using a monoexponential fit of signal intensity according to the following equation:

$$
\frac{S(b)}{S_{0}}=\exp (-b \mathrm{ADC})
$$

where $S(b)$ and $S_{0}$ are the diffusion-weighted signal intensity at a given $b$ value and $b=0 \mathrm{~s} / \mathrm{mm}^{2}$, respectively. The leastsquares method was used for linear fitting of the monoexponential model.

2.5. Statistical Analysis. Normally distributed and nonnormally distributed data were presented as the mean (SD) and median (IQR), respectively. DR, SNR, and CNR were compared between EPI-DWI and TSE-DWI using the Wilcoxon signed-rank test. The interprotocol reproducibility of ADC and LSR between the two sequences was assessed by calculating the 95\% Bland-Altman limits of agreement (LOAs). The ADC and LSR were evaluated using the Mann-Whitney test and receiver operating characteristic (ROC) analysis. Statistical analyses were carried out using SPSS (version 22.0, IBM Corporation, USA) and MedCalc software (version 18.2.1, Mariakerke, Belgium). A $P$ value $<0.05$ was considered statistically significant. 


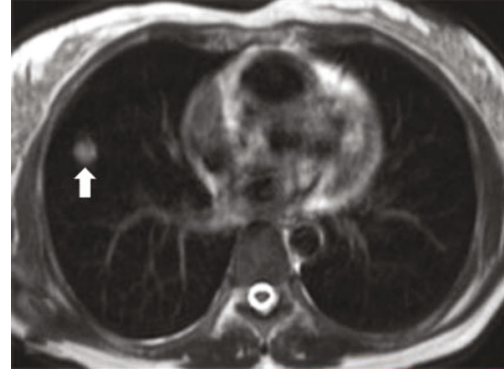

(a)

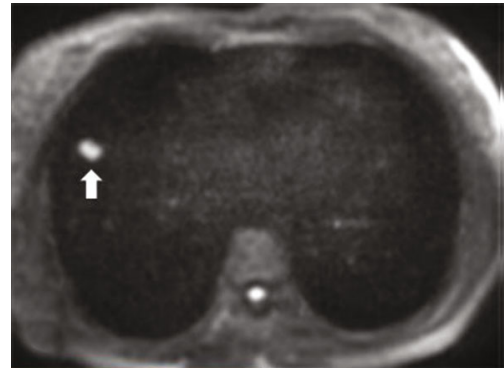

(c)

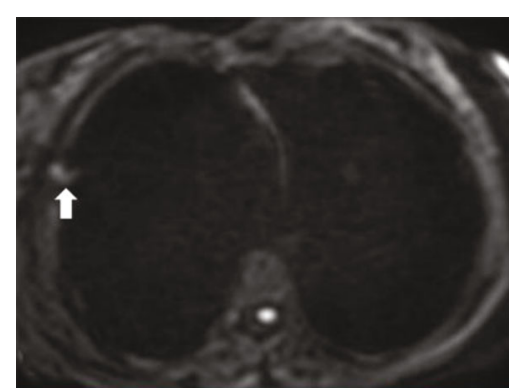

(b)

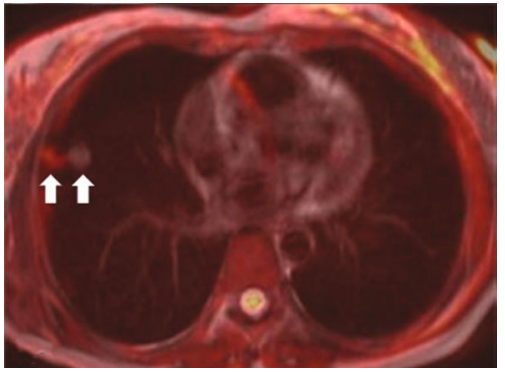

(d)

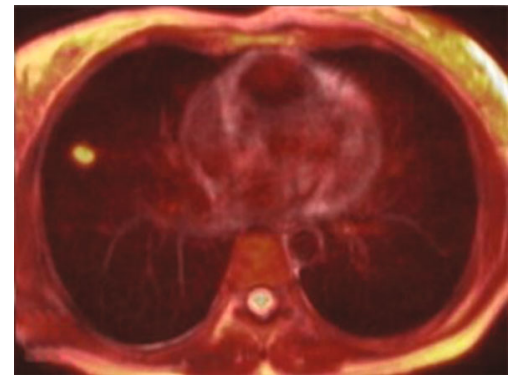

(e)

FIGURE 2: A nodular lesion (arrow) in the right middle lobe: (a) TSE-T2WI; (b) EPI-DWI $\left(b=600 \mathrm{~s} / \mathrm{mm}^{2}\right)$; (c) TSE-DWI $\left(b=600 \mathrm{~s} / \mathrm{mm}^{2}\right)$; (d) T2WI fused with EPI-DWI; (e) T2WI fused with TSE-DWI. The distortion of the lesion (double fine arrow) is illustrated on EPI-DWI, while the lesion on TSE-DWI demonstrates node formation or displacement compared to T2WI.

\section{Results}

3.1. Image Quality. Two representative fusion images, consisting of TSE-DWI on T2WI and EPI-DWI on T2WI, are shown in Figures 1 and 2, respectively. Figure 3 shows a contrast of DR, SNR, and CNR between EPI-DWI and TSE-DWI. Image distortion of DWI was significantly reduced in TSE-DWI as contrasted to EPI-DWI. The DR for SPLs in TSE-DWI and EPI-DWI was $0.000(0.029)$ and $0.224(0.483)$, respectively $(P<0.001)$. The mean SNR and CNR were slightly lower in TSE-DWI than those in EPI-DWI; however, no significant difference was detected between the two sequences.

3.2. Comparison of ADC and LSR between TSE-DWI and $E P I-D W I$. Figure 4 shows the Bland-Altman plots of ADC and LSR between EPI-DWI and TSE-DWI. The ADC and LSR of TSE-DWI exhibited slightly greater values than those of EPI-DWI in solitary pulmonary lesions (SPLs). The Bland-Altman analysis illustrated broad LOA between EPIDWI and TSE-DWI. The 95\% LOAs obtained were from $-64.00 \%$ to $62.00 \%$ for ADC and from $-156.00 \%$ to $100.00 \%$ for LSR.
3.3. Diagnostic Performance of Multiple Parameters. The ADC and LSR derived from EPI-DWI and TSE-DWI of malignant and benign SPLs are shown in Table 2, and the corresponding ROC curves for predicting malignant SPLs are plotted in Figure 5. The area under the curve (AUC) of ADC derived from TSE-DWI and EPI-DWI was 0.781 and 0.789 , respectively, and the corresponding cutoff value, sensitivity, and specificity were $1.450,81.82 \%$, and $72.73 \%$ and $1.380,86.36 \%$, and $81.82 \%$, respectively. Furthermore, the AUC of LSR derived from TSE-DWI and EPI-DWI were 0.748 and 0.818 , respectively, and the corresponding cutoff value, sensitivity, and specificity were $1.060,72.73 \%$, and $81.82 \%$ and $0.878,77.27 \%$, and $81.82 \%$, respectively (Table 3). However, no significant differences in diagnostic efficacy were observed among $\mathrm{ADC}_{(\mathrm{TSE}-\mathrm{DWI})}, \mathrm{ADC}_{(\mathrm{EPI}-\mathrm{DWI})}$, $\mathrm{LSR}_{(\mathrm{TSE}-\mathrm{DWI})}$, and LSR $\mathrm{LPI}_{\text {(EPWI) }}$.

\section{Discussion}

In the present study, we found that TSE-DWI provides distortion-free images with similar SNR and CNR compared with EPI-DWI. The diagnostic performance of ADC and LSR 

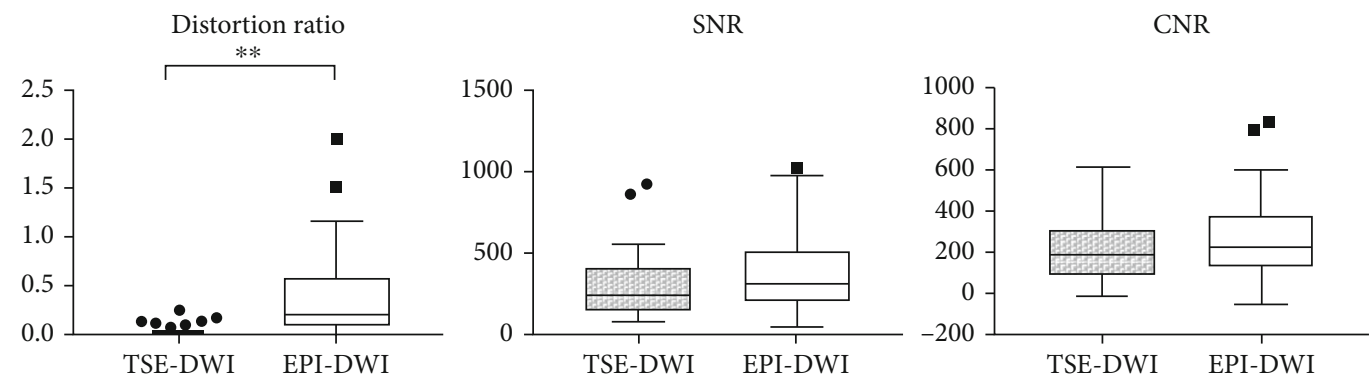

FIGURE 3: Comparison of distortion ratio (DR), signal-to-noise ratio (SNR), and contrast-to-noise ratio (CNR) between SS-TSE-DWI and SSEPI-DWI. Significant differences in DR were evaluated with a Wilcoxon signed-rank test. ** denotes $P<0.001$.
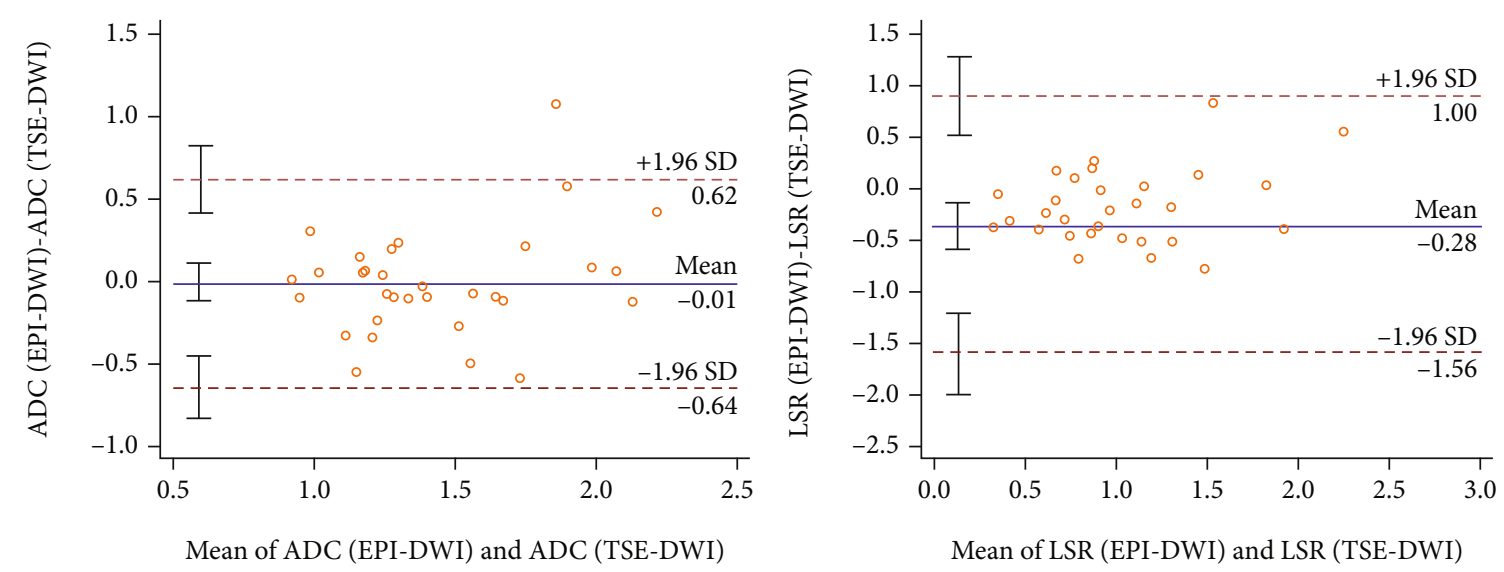

FIGURE 4: Bland-Altman plots for LSR and ADC of EPI-DWI and TSE-DWI. Continuous blue lines demonstrate mean differences, and dotted red lines show $95 \%$ limits of agreement (LOA).

TABLE 2: The mean ADC and LSR of TSE-DWI and EPI-DWI.

\begin{tabular}{|c|c|c|c|c|}
\hline Parameters & Benign & Malignant & $Z$ & $P$ \\
\hline \multicolumn{5}{|l|}{ TSE } \\
\hline $\operatorname{ADC}\left(\times 10^{-3} \mathrm{~mm}^{2} / \mathrm{s}\right)$ & $1.700(0.725)$ & $1.326(0.366)$ & -2.598 & 0.009 \\
\hline LSR & $0.757(0.354)$ & $1.304(0.780)$ & -2.291 & 0.022 \\
\hline \multicolumn{5}{|l|}{ EPI } \\
\hline $\operatorname{ADC}\left(\times 10^{-3} \mathrm{~mm}^{2} / \mathrm{s}\right)$ & $1.600(0.616)$ & $1.229(0.327)$ & -2.673 & 0.008 \\
\hline LSR & $0.603(0.386)$ & $1.073(0.471)$ & -2.941 & 0.003 \\
\hline
\end{tabular}

Note: data as median (IQR); Mann-Whitney test.

derived from TSE-DWI was as good as those from EPI-DWI. Additionally, LSR showed similar diagnostic performance compared to ADC.

The results of this study show that TSE-DWI may be an excellent substitute for EPI-DWI in patients undergoing lung examinations. As demonstrated by the DR comparison along the phase-encoding direction, free-breathing SS-TSE-DWI was superior to free-breathing EPI in minimizing geometric distortions. In TSE-DWI, the geometric precision was compared with that of a standard anatomic T2WI. Minimum distortion allowed easy registration and transfer of contours between T2WI and TSE-DWI. In EPI-DWI, a shift in the lesion position and distortion was often present at the lesion-air interface due to susceptibility artifacts.

The image noise lies in the voxel size, receiver bandwidth, and the total number of averages during image acquisition
[16]. In this study, the two DWI sequences had identical voxel sizes and number of averages. Although the larger inherent bandwidth of the TSE sequence could reduce SNR $[3,8]$, the SNR and CNR of the two sequences were identical; this finding is similar to a previous research [10].

The Bland-Altman analysis demonstrated broad LOAs for ADC (64\%) between EPI-DWI and TSE-DWI in our study, similar to previous studies [10, 17]. Moreover, we found that the LoA for LSR (up to $156.00 \%$ ) between the two sequences was even broader than that for ADC. This further confirms that the parameters of these two sequences cannot be directly substituted for each other. Consequently, the use of a specific quality assurance protocol as that proposed in the recent paper [18] is deeply recommended, in order to guarantee the clinical applications of the parameters of these two sequences. 


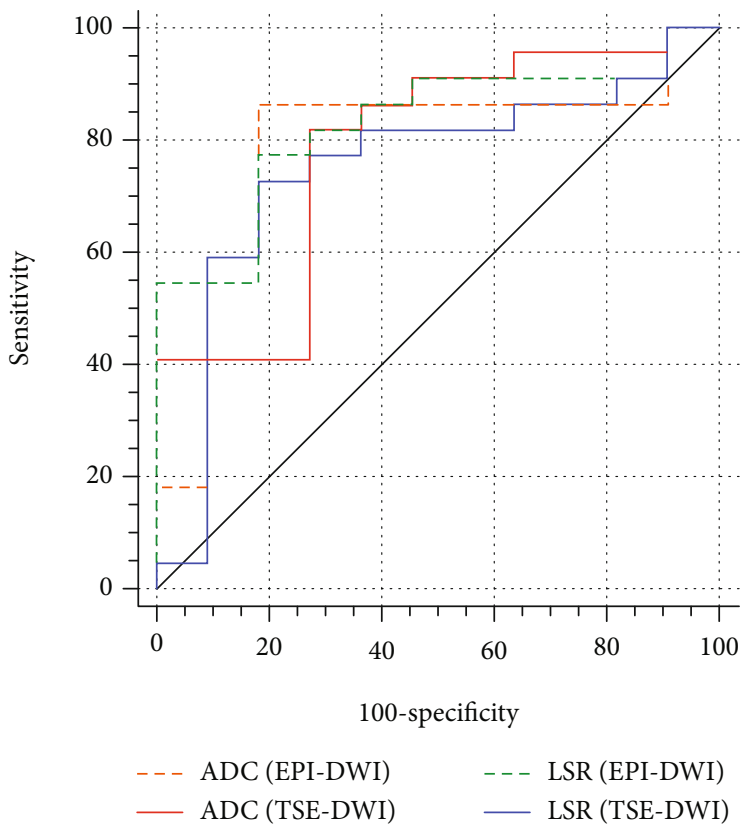

FIgURE 5: Results of receiver operating characteristic analysis for LSR and ADC derived from TSE- and EPI-DWI.

TABLE 3: Sensitivity and specificity of ADC and LSR at optimal cutoff values in differentiating malignant from benign solitary pulmonary lesions.

\begin{tabular}{|c|c|c|c|c|c|c|}
\hline Parameters & AUC & Cutoff value & Sensitivity (\%) & Specificity (\%) & $+\mathrm{LR}(\%)$ & -LR (\%) \\
\hline \multicolumn{7}{|l|}{ TSE } \\
\hline $\operatorname{ADC}\left(\times 10^{-3} \mathrm{~mm}^{2} / \mathrm{s}\right)$ & 0.781 & 1.450 & 81.82 & 72.73 & 3.00 & 0.25 \\
\hline LSR & 0.748 & 1.060 & 72.73 & 81.82 & 4.00 & 0.33 \\
\hline \multicolumn{7}{|l|}{$E P I$} \\
\hline $\operatorname{ADC}\left(\times 10^{-3} \mathrm{~mm}^{2} / \mathrm{s}\right)$ & 0.789 & 1.380 & 86.36 & 81.82 & 4.75 & 0.17 \\
\hline LSR & 0.818 & 0.878 & 77.27 & 81.82 & 4.25 & 0.28 \\
\hline
\end{tabular}

Note: AUC: area under the ROC curve; ADC: apparent diffusion coefficient; LSR: lesion-to-spinal cord signal intensity ratio; +LR: positive likelihood ratio; -LR: negative likelihood ratio.

In our study, we found that both EPI-DWI and TSEDWI can distinguish malignant from benign SPLs according to ADC and LSR. The ADC in the malignant was lower than that in benign lesions, and LSR showed the opposite result. This is because malignant lesions tend to have a higher cell density and narrower extracellular space, which hinders the diffusion movement of water molecules [19]. Besides, we compared LSR and ADC from both sequences in the differentiation of SPLs and found no significant difference between them. Our results are consistent with some studies $[13,20]$, but not the same as others $[12,21]$. These inconsistent results are likely to be attributed to the bias of the included cases, lesion size, variance in imaging quality, and the reduced sample size.

Unexpectedly, even with less image distortion, the quantitative parameters of ADC and LSR derived from TSE-DWI are not better than (or even slightly second to) EPI-DWI in terms of diagnostic accuracy. LSR DWI) showed the lowest diagnostic efficiency, although the difference among these parameters was not significant.
One possible explanation is that ROI was typically placed at the location with the highest signal intensity during assessing LSR. The acquisition time of TSE-DWI is longer, and its blurring effect may affect the ROI delineation and interpretation of lesions' signal intensity. This should be confirmed in future studies.

This study had some limitations. First, the image acquisition times of EPI-DWI and TSE-DWI were not the same. Therefore, the SNR per unit time was not the same. We did not increase the acquisition time of EPI to the same as that of TSE, which is what we are going to do in our following studies; this could lead to a significantly higher SNR and CNR for EPI compared to TSE sequences. Second, the population of this study was relatively small. Some other benign (e.g., round atelectasis) and malignant (e.g., carcinoid and single metastasis) lesions were not included in this study. Furthermore, only nodules larger than $8 \mathrm{~mm}$ in diameter and pathologically confirmed were included; the inclusion of all nodules encountered in clinical practice might yield different results. 
In conclusion, TSE-DWI can be an excellent alternative to EPI-DWI for imaging pulmonary lesions sensitive to distortion. Despite less image distortion, the quantitative parameters of ADC and LSR from TSE-DWI demonstrate similar diagnostic accuracy for the differentiation of SPLs as compared with EPI-DWI.

\section{Data Availability}

All the materials and data in studies are available.

\section{Ethical Approval}

All procedures performed in studies involving human participants were in accordance with the ethical standards of the institutional and/or national research committee and with the 1964 Helsinki declaration and its later amendments or comparable ethical standards. This retrospective study was approved by the medical ethical committee of Guangdong Second Provincial General Hospital.

\section{Consent}

Informed written consent was obtained.

\section{Conflicts of Interest}

None of the authors have a conflict of interest.

\section{Authors' Contributions}

Qiang Lei and Qi Wan contributed equally to this work.

\section{Acknowledgments}

This study was funded by the Youth Foundation of Guangdong Second Provincial General Hospital (grant number YQ2019-004) and the National Natural Science Foundation of China (grant number 81601457).

\section{References}

[1] S. Ahlawat and L. M. Fayad, "Diffusion weighted imaging demystified: the technique and potential clinical applications for soft tissue imaging," Skeletal Radiology, vol. 47, no. 3, pp. 313-328, 2018.

[2] M. M. Khoo, P. A. Tyler, A. Saifuddin, and A. R. Padhani, "Diffusion-weighted imaging (DWI) in musculoskeletal MRI: a critical review," Skeletal Radiology, vol. 40, no. 6, pp. 665$681,2011$.

[3] N. Tyagi, M. Cloutier, K. Zakian, J. O. Deasy, M. Hunt, and A. Rimner, "Diffusion-weighted MRI of the lung at 3T evaluated using echo-planar-based and single-shot turbo spinecho-based acquisition techniques for radiotherapy applications," Journal of applied clinical medical physics, vol. 20, no. 1, pp. 284-292, 2019.

[4] B. De Foer, J. P. Vercruysse, B. Pilet et al., "Single-shot, turbo spin-echo, diffusion-weighted imaging versus spin-echo-planar, diffusion-weighted imaging in the detection of acquired middle ear cholesteatoma," AJNR American journal of neuroradiology, vol. 27, no. 7, pp. 1480-1482, 2006.
[5] K. Kamimura, M. Nakajo, Y. Fukukura et al., "Intravoxel incoherent motion in normal pituitary gland: initial study with turbo spin-echo diffusion-weighted imaging," AJNR American journal of neuroradiology, vol. 37, no. 12, pp. 2328-2333, 2016.

[6] A. Hiwatashi, O. Togao, K. Yamashita et al., "Diffusivity of intraorbital lymphoma vs. inflammation: comparison of single shot turbo spin echo and multishot echo planar imaging techniques," European radiology, vol. 28, no. 1, pp. 325-330, 2018.

[7] J. Sakamoto, Y. Sasaki, M. Otonari-Yamamoto, and T. Sano, "Comparison of various methods for quantification of apparent diffusion coefficient of head and neck lesions with HASTE diffusion-weighted MR imaging," Oral surgery, oral medicine, oral pathology and oral radiology, vol. 114, no. 2, pp. 266276, 2012.

[8] K. Hirata, T. Nakaura, T. Okuaki et al., "Comparison of the image quality of turbo spin echo- and echo-planar diffusionweighted images of the oral cavity," Medicine, vol. 97, no. 19, p. e0447, 2018.

[9] W. Panyarak, T. Chikui, Y. Yamashita, T. Kamitani, and K. Yoshiura, "Image Quality and ADC Assessment in Turbo Spin-Echo and Echo-Planar Diffusion- Weighted MR Imaging of Tumors of the Head and Neck," Academic radiology, vol. 26, no. 10, pp. e305-e316, 2019.

[10] Q. Wan, Q. Lei, P. Wang et al., "Intravoxel incoherent motion diffusion-weighted imaging of lung cancer: comparison between turbo spin-echo and echo-planar imaging," Journal of computer assisted tomography, vol. 44, no. 3, pp. 334-340, 2020.

[11] G. Shen, Z. Jia, and H. Deng, "Apparent diffusion coefficient values of diffusion-weighted imaging for distinguishing focal pulmonary lesions and characterizing the subtype of lung cancer: a meta-analysis," European radiology, vol. 26, no. 2, pp. 556-566, 2016.

[12] T. Uto, Y. Takehara, Y. Nakamura et al., "Higher sensitivity and specificity for diffusion-weighted imaging of malignant lung lesions without apparent diffusion coefficient quantification," Radiology, vol. 252, no. 1, pp. 247-254, 2009.

[13] N. Henz Concatto, G. Watte, E. Marchiori et al., "Magnetic resonance imaging of pulmonary nodules: accuracy in a granulomatous disease-endemic region," European radiology, vol. 26, no. 9, pp. 2915-2920, 2016.

[14] V. Cakmak, F. Ufuk, and N. Karabulut, "Diffusion-weighted MRI of pulmonary lesions: comparison of apparent diffusion coefficient and lesion-to-spinal cord signal intensity ratio in lesion characterization," Journal of magnetic resonance imaging : JMRI, vol. 45, no. 3, pp. 845-854, 2017.

[15] G. Shen, H. Ma, B. Liu, P. Ren, and A. Kuang, "Diagnostic performance of DWI with multiple parameters for assessment and characterization of pulmonary lesions: a meta-analysis," AJR American journal of roentgenology, vol. 210, no. 1, pp. 58-67, 2018.

[16] J. T. Heverhagen, "Noise measurement and estimation in MR imaging experiments," Radiology, vol. 245, no. 3, pp. 638639, 2007.

[17] R. Mikayama, H. Yabuuchi, S. Sonoda et al., "Comparison of intravoxel incoherent motion diffusion-weighted imaging between turbo spin-echo and echo-planar imaging of the head and neck," European Radiology, vol. 28, no. 1, pp. 316-324, 2018.

[18] L. Fedeli, G. Belli, A. Ciccarone et al., "Dependence of apparent diffusion coefficient measurement on diffusion gradient direction and spatial position - A quality assurance 
intercomparison study of forty-four scanners for quantitative diffusion-weighted imaging," Physica Medica, vol. 55, pp. 135-141, 2018.

[19] Q. Wan, Y. S. Deng, Q. Lei et al., "Differentiating between malignant and benign solid solitary pulmonary lesions: are intravoxel incoherent motion and diffusion kurtosis imaging superior to conventional diffusion-weighted imaging?," European radiology, vol. 29, no. 3, pp. 1607-1615, 2019.

[20] H. X. Guan, Y. Y. Pan, Y. J. Wang, D. Z. Tang, S. C. Zhou, and L. M. Xia, "Comparison of various parameters of DWI in distinguishing solitary pulmonary nodules," Current medical science, vol. 38, no. 5, pp. 920-924, 2018.

[21] H. Koyama, Y. Ohno, S. Seki et al., "Value of diffusionweighted MR imaging using various parameters for assessment and characterization of solitary pulmonary nodules," European journal of radiology, vol. 84, no. 3, pp. 509-515, 2015. 